\title{
Variation of the density of states in amorphous GdSi at the metal-insulator transition
}

\author{
L. Bokacheva, ${ }^{1}$ W. Teizer, ${ }^{2}$ F. Hellman, ${ }^{1}$ and R. C. Dynes ${ }^{1}$ \\ ${ }^{1}$ Department of Physics, University of California, San Diego, La Jolla, California 92093-0319, USA \\ ${ }^{2}$ Department of Physics, Texas A\&M University, College Station, Texas 77843-4242, USA \\ (Received 26 September 2003; revised manuscript received 1 March 2004; published 29 June 2004)
}

\begin{abstract}
We have performed detailed conductivity and tunneling measurements on the amorphous, magnetically doped material $\alpha-\mathrm{Gd}_{x} \mathrm{Si}_{1-x}$, which can be driven through the metal-insulator transition by the application of an external magnetic field. Low temperature conductivity increases linearly with field near the transition and slightly slower on the metallic side. The tunneling conductance, proportional to the density of states $N(E)$, undergoes a gradual change with increasing field, from insulating, showing a soft gap at low bias, with a slightly slower than parabolic energy dependence, i.e., $N(E) \sim E^{c}, c \lesssim 2$, towards metallic behavior, with $E^{d}$, $0.5<d<1$ energy dependence. The density of states at the Fermi level in a sample, which shows already a small, but metal-like conductivity, appears to be zero at low fields, as in an insulator. We speculate that this behavior is due to the microscopic percolation nature of transport near the transition.
\end{abstract}

DOI: 10.1103/PhysRevB.69.235111

PACS number(s): 71.23.Cq, 75.50.Pp, 71.30.+h

\section{INTRODUCTION}

The metal-insulator transition (MIT) in disordered systems ${ }^{1}$ is a complex problem, complete and accurate treatment of which requires that the effects of localization and the electronic correlations be taken into account simultaneously and on equal footing. The carriers localized by the disordered potential cannot screen their interactions as well as the mobile ones, and therefore the Coulomb interactions among them are strong and cannot be treated perturbatively.

Insulating and metallic behaviors are defined as follows. The sample is an insulator if its conductivity $\sigma(T)$ extrapolated to zero temperature vanishes: $\sigma(T=0)=0$. If $\sigma(T=0)$ is finite, the sample is on the metallic side of the transition. Far from the transition, theoretical models have successfully described the effects due to the interactions in the conductivity $\sigma$ and the density of states (DOS) $N(E)$. The transport conductivity of a metallic material with strong Coulomb correlations follows a power law as a function of temperature: $\sigma(T)=\sigma_{0}+\sigma_{1} T^{y}$, with $y=0.5 .^{2}$ On the insulating side, the interactions modify the exponent of the temperature dependence of the variable range hopping (VRH) conductivity from $1 / 4$ in the noninteracting case $^{3}$ to $1 / 2: \sigma(T) \sim \exp [$ $\left.-\left(T_{0} / T\right)^{1 / 2}\right] .{ }^{4}$ In the density of states, on either side of the MIT, interactions generally cause the depletion of states near the Fermi energy. In the metallic regime the correlations are manifested as a square-root dip in the DOS: $N(E)=N(0)[1$ $\left.+(E / \Delta)^{1 / 2}\right]$, where $\Delta \sim \hbar D / l^{2}$ is the correlation energy, $D$ is the diffusion coefficient, $l$ is the mean free path, and $\hbar$ is Planck's constant. ${ }^{5,6}$ In an insulator with thermally activated VRH conductivity, the interactions lead to the opening of a soft gap with a quadratic [in the three-dimensional (3D) case] energy dependence, $N(E)=(3 / \pi)\left(\kappa / e^{2}\right)^{3} E^{2}$, where $\kappa$ is the dielectric constant and $e$ is the electronic charge. The width of the Coulomb gap $\Delta_{c}$ is determined by $\kappa$ and the noninteracting density of states $N_{0}: \Delta_{c}=e^{3}\left(N_{0} / \kappa^{3}\right)^{1 / 2}$. ${ }^{7}$

The presence of the features due to the Coulomb interactions in the conductivity and DOS in disordered systems near the MIT has been well established experimentally. These ef- fects have been mostly observed in experiments performed using the discrete sets of crystalline ${ }^{8}$ and amorphous samples ${ }^{9}$ with different dopant concentrations close to the critical one.

Amorphous systems have an advantage over the crystalline materials for the MIT studies since they undergo the transition at doping concentrations that are orders of magnitude higher than those in the crystalline materials due to the increased disorder (roughly $x \sim 10^{-1}$ in amorphous versus $x$ $\sim 10^{-5}$ in crystalline materials). The Fermi temperature $T_{F}$ of amorphous systems is therefore much higher than in the crystalline ones, and $T / T_{F}$ is much lower. For amorphous systems the region where the Ioffe-Regel localization criterion ${ }^{10} k_{F} l \sim 1$ is valid ( $k_{F}$ is the Fermi wave vector) extends to much higher values of the conductivity: the maximum critical conductivity $\sigma=n e^{2} /\left(\hbar k_{F}^{2}\right)$, where $n$ is the concentration, reaches $500(\Omega \mathrm{cm})^{-1}$ at $n \sim 10^{22} \mathrm{~cm}^{-3}$, as opposed to $20(\Omega \mathrm{cm})^{-1}$ in the doped crystalline semiconductors. ${ }^{11}$ Therefore in amorphous materials one can probe the transition deeper in the critical regime at accessible temperatures.

In some materials it is possible to observe the MIT in a single sample and tune it by varying an external parameter, such as magnetic field, ${ }^{12}$ stress, ${ }^{13}$ or illumination. ${ }^{14}$ This approach eliminates undesirable scatter in the sample parameters and thus is preferable to studying a discrete set of samples. For this reason, magnetically doped amorphous materials, such as $\alpha-\mathrm{Gd}_{x} \mathrm{Si}_{1-x}(\mathrm{GdSi})$ and $\mathrm{Tb}_{x} \mathrm{Si}_{1-x},{ }^{15,16}$ are particularly suited for the studies of the MIT. In these materials besides the structural disorder, there is an additional degree of disorder associated with random orientation of the ionic magnetic moments, which can be controlled by the applied magnetic field. When the magnetic moments of the impurity ions are aligned by the external field, the disorder is reduced, and the mobility edge $E_{c}$ is lowered. ${ }^{17}$ This promotes delocalization of carriers and increases the conductivity of the material. Thus a sample doped slightly below the critical level at low temperature (below $50 \mathrm{~K}$ ) shows a large negative magnetoresistance and can be continuously driven 
through the transition by magnetic field. It is also possible to probe the density of states of these materials simultaneously with their transport properties by studying the tunneling of quasiparticles through a thin oxide barrier between the material in question and a metallic electrode. These experiments enable one to track the changes that occur in the DOS near the MIT and to separate them from the mobility effects, which also contribute to the conductivity.

In GdSi the MIT occurs at $x \approx 0.14$ of $\mathrm{Gd}$ in zero magnetic field. Near the critical doping level, $\sigma(T=0)$ is very sensitive to the slight variations of $x$. This material has been extensively studied: its structure, ${ }^{18}$ transport conductivity, ${ }^{15,19,20}$ tunneling conductance, ${ }^{17,21}$ specific heat ${ }^{22}$ magnetization, ${ }^{23}$ infrared absorption, ${ }^{24}$ spin polarization, ${ }^{25}$ and the Hall effect, ${ }^{26}$ have been investigated in detail. It has been shown that the magnetic field increases the conductivity while raising both the concentration of the free carriers and the density of states. ${ }^{17}$ The density of free carriers has been found to vary linearly with magnetic field. ${ }^{26}$

The results of the tunneling measurements ${ }^{17}$ indicate that for metallic $\mathrm{GdSi}$ samples with $\sigma_{0}$ still below the Ioffe-Regel condition, the DOS has $E^{1 / 2}$ dependence with a nonvanishing $N(0)$. The tunneling conductance curves $d I / d V(V)$ at different constant magnetic fields are approximately parallel to each other with only $N(0)$ increasing with field. On the insulating side these experiments are limited by the increasing resistance of the GdSi film. When the resistance of the insulating GdSi film over the area of the tunnel junction becomes comparable to the resistance of the junction itself, the voltage drop occurs both over the junction and the film, so that the bias voltage cannot be considered a true energy coordinate. In samples which become conducting in magnetic field, the DOS shows a soft gap near zero bias with somewhat weaker than $E^{2}$ dependence and $N(0)$ vanishing at low fields. The tunneling data also suggest that in the transition region the DOS evolves continuously from insulating, showing a soft gap at zero bias, to metallic, with a square root cusp, upon increasing magnetic field. The DOS at the Fermi level $N(0)$ has been shown to depend on magnetic field as $(H$ $\left.-H_{c}\right)^{2}$ near the transition. ${ }^{17}$ The models for the DOS described above are valid far from the transition, yet cannot be applied in the critical region or properly merged at the critical concentration. The renormalization group techniques ${ }^{27,28}$ in the critical region provide formal mathematical treatment, which cannot be easily applied to the description of tunneling experiments. It is not known how the DOS evolves from the insulating form to the metallic one. It is therefore desirable to be able to tune a single sample through the MIT and measure its transport and tunneling properties as close to the transition as possible.

\section{EXPERIMENTAL RESULTS}

In this paper we focus on the region in the immediate vicinity of the MIT and investigate the simultaneous variation of the transport conductivity and the DOS in a detailed and systematic way. The results presented here were obtained using $\mathrm{GdSi}$ samples prepared as described previously. ${ }^{17}$ Amorphous films of GdSi, 100-nm-thick, have been grown

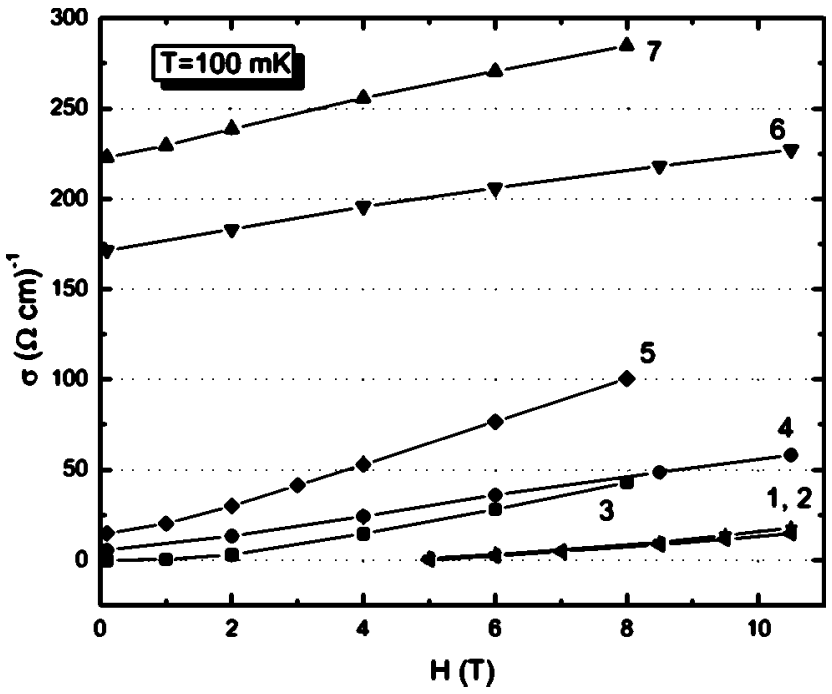

FIG. 1. Low temperature $(T=100 \mathrm{mK})$ conductivity $\sigma(H)$ of seven different samples vs the applied magnetic field. At $H=0.1 \mathrm{~T}$ samples No. 1 and No. 2 are insulating, sample No. 3 is at the transition, samples No. 4 and No. 5 are slightly metallic, and samples No. 6 and No. 7 are in the metallic regime.

by electron-beam coevaporation. Tunnel junctions were formed by an insulating barrier of the native GdSi oxide and $\mathrm{Pb}$ counterelectrodes. The tunnel junctions also served as voltage terminals in the four point transport measurements. We selected only those samples for which the junction resistance was at least an order of magnitude higher than the film resistance over the area of the junction.

\section{A. Transport conductivity measurements}

We use the actual conductivity values at low temperature $(T=100 \mathrm{mK})$ in order to determine the sample's proximity to the MIT and avoid the ambiguity associated with the extrapolation of $\sigma(T)$ to $T=0$. Figure 1 shows the low temperature conductivity versus the applied magnetic field for several different samples, with the conductivity spanning a broad range from zero to over $200(\Omega \mathrm{cm})^{-1}$, all below the Ioffe-Regel limit. The relative increase of the conductivity with field is the largest in the most insulating samples No. 1 and No. 2. Here the conductivity is a faster than linear function of the applied field and increases from nearly zero at $4-5 \mathrm{~T}$ to $14-17(\Omega \mathrm{cm})^{-1}$ at $H=10.5 \mathrm{~T}$, i.e., by a factor of 40 or 50. In samples which are at the transition or barely on the metallic side, Nos. 3-5, the conductivity at a constant low temperature increases approximately linearly with magnetic field. In sample No. 3, which is slightly insulating, the conductivity grows between 0.1 and $8 \mathrm{~T}$ by a factor of 40 , but only by a factor of 10 for slightly metallic samples No. 4 and No. 5. Conductivity of the most metallic samples No. 6 and No. 7 is a slightly sublinear function of the field and increases only by about $25 \%$ of its value at $H=0.1 \mathrm{~T}$.

The conductivity versus temperature curves for the barely metallic sample No. $5\left[\sigma(50 \mathrm{mK})=14(\Omega \mathrm{cm})^{-1}\right]$, obtained from the dc $I V$ transport measurements at constant fields from 0.1 to $8 \mathrm{~T}$, are shown in Fig. 2. The dc measurement 


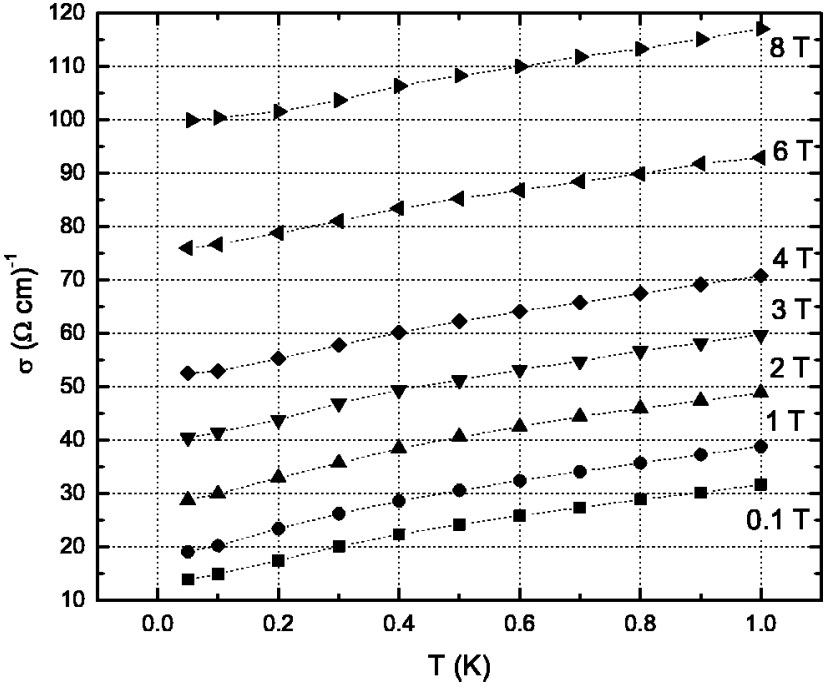

FIG. 2. Conductivity of a slightly metallic GdSi sample (sample No. 5) vs temperature.

technique is more appropriate in this case because at low temperatures near the MIT the resistance and the dielectric constant of GdSi films are strongly frequency dependent. As shown before, ${ }^{17}$ the conductivity curves can be fit with $\sigma(T)=\sigma_{0}+\sigma_{1} T^{1 / 2}+\sigma_{2} T$, as predicted for a metal with correlations. The curves at different fields are approximately parallel to each other, only shifted towards higher values, which implies that $\sigma_{0}$ grows roughly linearly with field, as illustrated in Fig. 1, and $\sigma_{1}$ is weakly dependent on $H$. This can be easily seen in Fig. 3, which shows the same data as in Fig. 2 , plotted as a function of $T^{1 / 2}$. The lowest temperature offset $\sigma_{0}$, in this case determined from the linear fits to $\sigma\left(T^{1 / 2}\right)$, has been subtracted from each curve. The linear variation of $\sigma_{0}$ with field is shown in the inset of Fig. 3. The $\sigma\left(T^{1 / 2}\right)$ data at different fields overlap well and, except at the lowest tem-

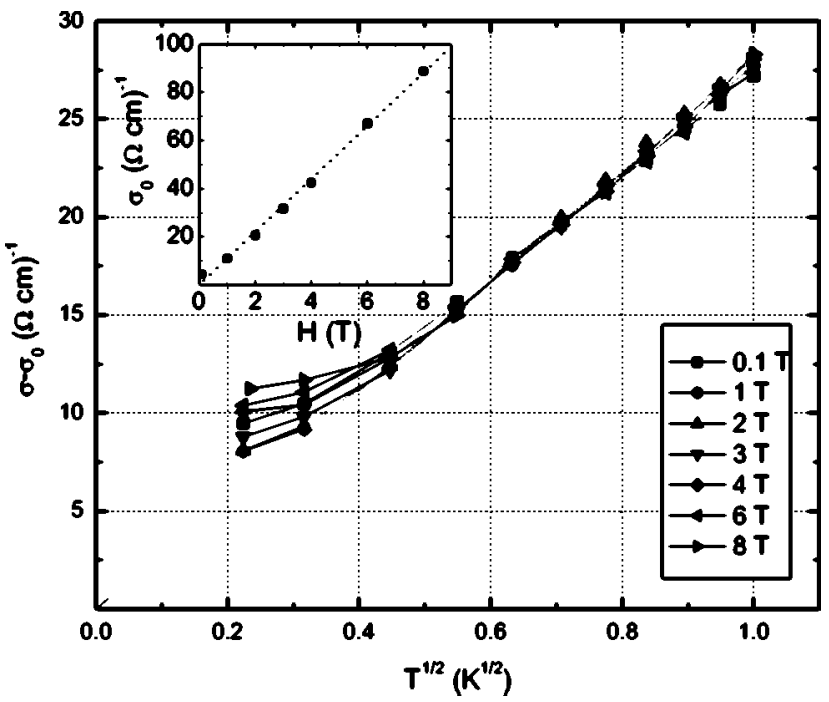

FIG. 3. Conductivity $\sigma-\sigma_{0}$ from the data in Fig. 2 (sample No. 5) vs $T^{1 / 2}$ with $\sigma_{0}$ determined from linear fits to $\sigma\left[\left(T^{1 / 2}\right)\right]$ subtracted. The solid line is a linear fit to the data. Inset: $\sigma_{0}$ vs magnetic field $H$. The dotted line is a linear fit.

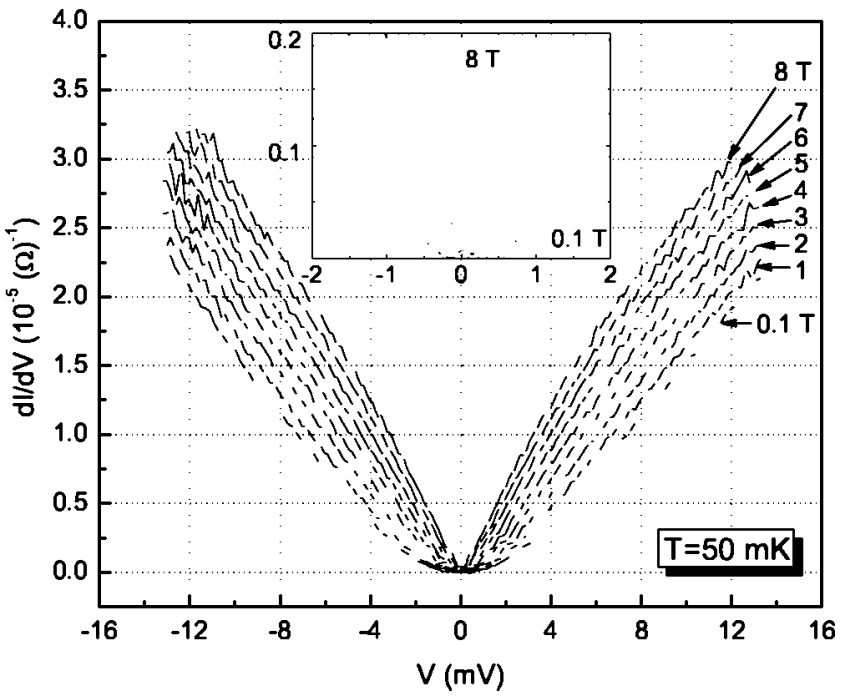

FIG. 4. Tunneling conductance $d I / d V(V)$ curves of sample No. 5 at $T=50 \mathrm{mK}$ and different magnetic fields. Inset: Same data in the low bias region. The axes are in the same units as the main figure.

peratures, fall onto a straight line. The lowest temperature deviation may be due to the thermal or noise broadening. This dependence has been observed in nonmagnetic materials, which supports the idea that the main effect of the applied magnetic field is to decrease the disorder and lower the mobility edge, thus increasing the conductivity and driving the sample further into the metallic regime.

\section{B. Tunneling conductance measurements}

To elucidate the crossover in the DOS from the insulating to the metallic behavior we performed detailed tunneling studies. The data presented below were obtained as the dc $I V$ curves measured at constant magnetic fields and a constant low temperature and then numerically differentiated. Figure 4 shows a set of the differential tunneling conductance curves $d I / d V(V)$ for sample No. 5 at $50 \mathrm{mK}$ and magnetic fields from 0.1 to $8 \mathrm{~T}$.

At the lowest field $H=0.1 \mathrm{~T}$ (required to quench the superconductivity of the $\mathrm{Pb}$ counterelectrodes) the tunneling conductance curve is superlinear in the whole range of the bias voltage between $\pm 12 \mathrm{mV}$, but cannot be fit with a simple function. Near zero bias at $|V|<2.5 \mathrm{mV}$ the curve can be fit with a power law $a+b|V|^{c}$, with $c \sim 1.72$, close to the $V^{2}$ dependence expected of the fully formed Coulomb gap. Outside this region the exponent $c$ is only slightly greater than unity: $c \sim 1.1$. At $V=0$ the curve touches the horizontal axis, i.e., the zero bias conductance is unmeasurably small: $d I / d V(V=0)=0$. Upon increasing the field, the curves undergo a gradual and continuous change. The gap region with $c \gtrsim 1.5$ contracts and by $8 \mathrm{~T}$ it exists only in a small interval at $|V|<0.6 \mathrm{mV}$. The approximately linear part of the curve at higher bias expands and at the same time the exponent $c$ in this region decreases from nearly unity at $0.1 \mathrm{~T}$ to 0.74 at $8 \mathrm{~T}$, approaching the $V^{1 / 2}$ behavior seen in the clearly metallic samples. Starting at about $H=2 \mathrm{~T}$, the $d I / d V$ curves have an inflection point, separating the two parts of the curve with 


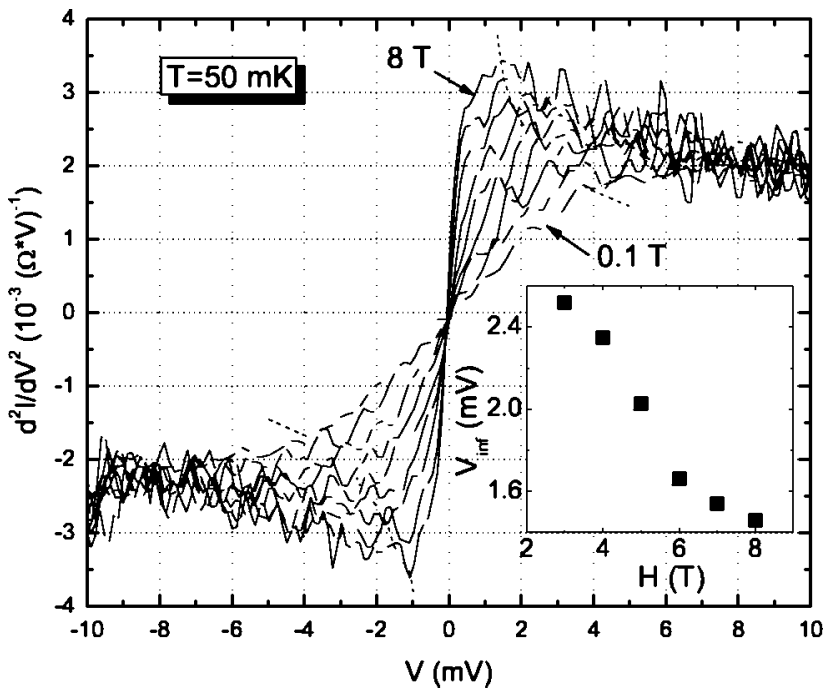

FIG. 5. Second derivative of the tunneling $I V, d^{2} I / d V^{2}(V)$ for the data in Fig. 4 (sample No. 5). The dashed line is a guide for the eye indicating the position of a maximum shifting to lower bias with increasing field. Inset: The bias voltage $V_{\text {inf }}$ at which the inflection point in the $d I / d V$ curves is located, plotted vs magnetic field.

$c>1$ (positive curvature), implying a "soft Coulomb gap," and $c<1$ (negative curvature). At the highest field $H=8 \mathrm{~T}$, the $d I / d V$ curve is sublinear almost in the entire range of the bias voltage and is characteristic of the metallic regime.

This continuous crossover can be seen in more detail in the inset of Fig. 4, which shows a zoom-in of the data in the low bias region below $2 \mathrm{mV}$. Zero bias conductance (ZBC) appears to be zero at $H=0.1$ and $1 \mathrm{~T}$, but increases at higher fields.

The gradual change in the shape of the $d I / d V(V)$ curves and the position of the inflection point can be easily seen in a plot of the second derivative of the tunneling $I V$ curves, $d^{2} I / d V^{2}(V)$, shown in Fig. 5. The $d^{2} I / d V^{2}$ curves reflect the curvature of the $I V$ and the slope of the $d I / d V$ curves. The maxima and minima of $d^{2} I / d V^{2}$ correspond to the inflection points in $d I / d V$, where the $d I / d V$ curvature changes from positive to negative. Since the $d I / d V$ curves are symmetric, we will refer only to the positive bias part of the data. At the lowest fields, 0.1 and $1 \mathrm{~T}$, the $d^{2} I / d V^{2}$ curves do not have a maximum, although their slope decreases significantly at 3.75 and $3.6 \mathrm{mV}$, respectively, and falls almost to zero above $6 \mathrm{mV}$. The maximum, i.e., the inflection point in $d I / d V$, appears at $H=2 \mathrm{~T}$ and at higher fields becomes more pronounced and moves towards zero bias. The shift of the voltage position of the inflection point with magnetic field is shown in the inset of Fig. 5.

These data, we suggest, indicate that the crossover from insulating $V^{2}$ behavior to metallic $V^{1 / 2}$ behavior is continuous. We cannot identify from the tunneling data a "critical point" that marks the transition. We have observed similar behavior in the tunneling characteristics of all samples studied to date in the vicinity of the MIT. In fact, the $d I / d V(V)$ data for different samples can be scaled to form one "master set" of curves, progressively changing their shape from insulating to metallic, correlated with the sample conductivity, as

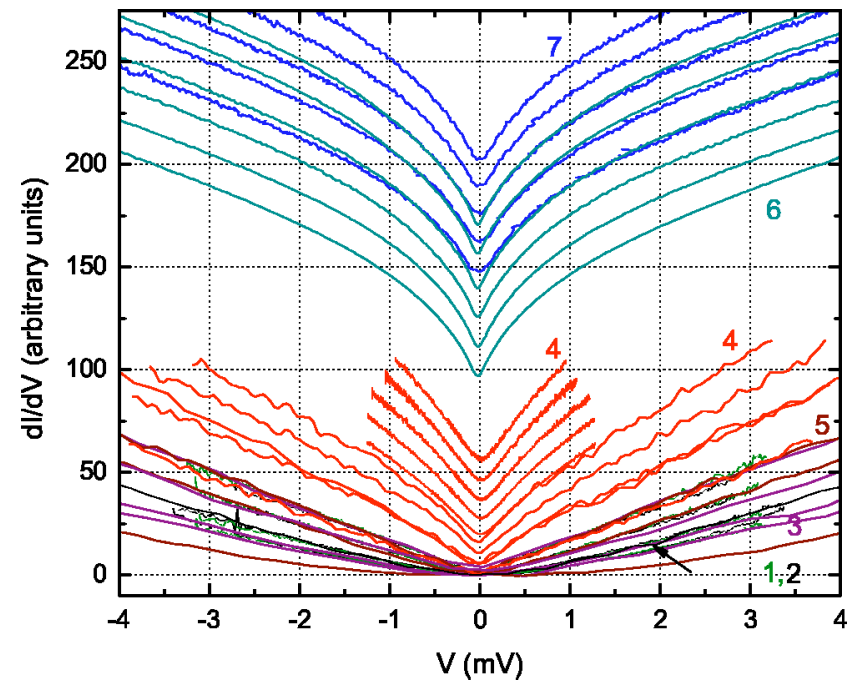

FIG. 6. (Color online) Rescaled tunneling conductance vs bias voltage at different magnetic fields for all samples at $T=100 \mathrm{mK}$, showing progressive change of bias dependence from insulating $\left(V^{2}\right)$ to metallic $\left(V^{1 / 2}\right)$.

shown in Fig. 6. The data for all samples have been taken as dc $I V$ curves and for sample No. 4 also as ac $d I / d V(V)$ measurements at fields up to $18 \mathrm{~T}$, performed at NHMFL (Tallahassee, Florida). These ac data are shown as a set of curves in a smaller bias voltage interval between approximately $\pm 1 \mathrm{mV}$. Each sample's data have been rescaled by a numerical factor. The curves of samples No. 1-5 overlap well and merge into a single set of characteristics, starting with superlinear (insulating) behavior at low fields and evolving towards the sublinear (metallic) behavior. Sample No. 4, which is the closest to the MIT and for which the data in a wide field range are available, shows both types of behavior. At low fields its $d I / d V$ curves overlap with those of more insulating samples No. 1-3 and at high fields they become similar to the curves of a substantially more conductive sample, No. 6. Samples No. 6 and 7 show the metallic $d I / d V$ curves at all fields, but their tunneling characteristics also change with increasing field, although less significantly than those of samples No. 1-5. The curves of samples No. 6 and 7 follow $V^{a}$ dependence, where $a$ decreases slightly with field from 0.7 at $0.1 \mathrm{~T}$ to approximately 0.57 at $8-10.5 \mathrm{~T}$, which we interpret as approaching typical metallic $V^{1 / 2}$ behavior. The combined $d I / d V$ data suggest that the gradual variation of the tunneling conductance is a general tendency, which is observed consistently and reproducibly in a set of samples.

The variation of the low temperature conductivity and zero bias tunneling conductance for all samples in the critical region is illustrated in Fig. 7. The conductivity data have been normalized by their values (measured or extrapolated) at $10.5 \mathrm{~T}$. The zero bias conductance data for each sample, which reflect the arbitrary resistance of the oxide barrier of the tunnel junction, have been rescaled and also normalized to its high field values. Note that in samples No. 3-5, which at low fields show a small, but metallic transport conductivity, the tunneling zero bias conductance appears to be unmeasurably small at the same fields. In these samples the insu- 

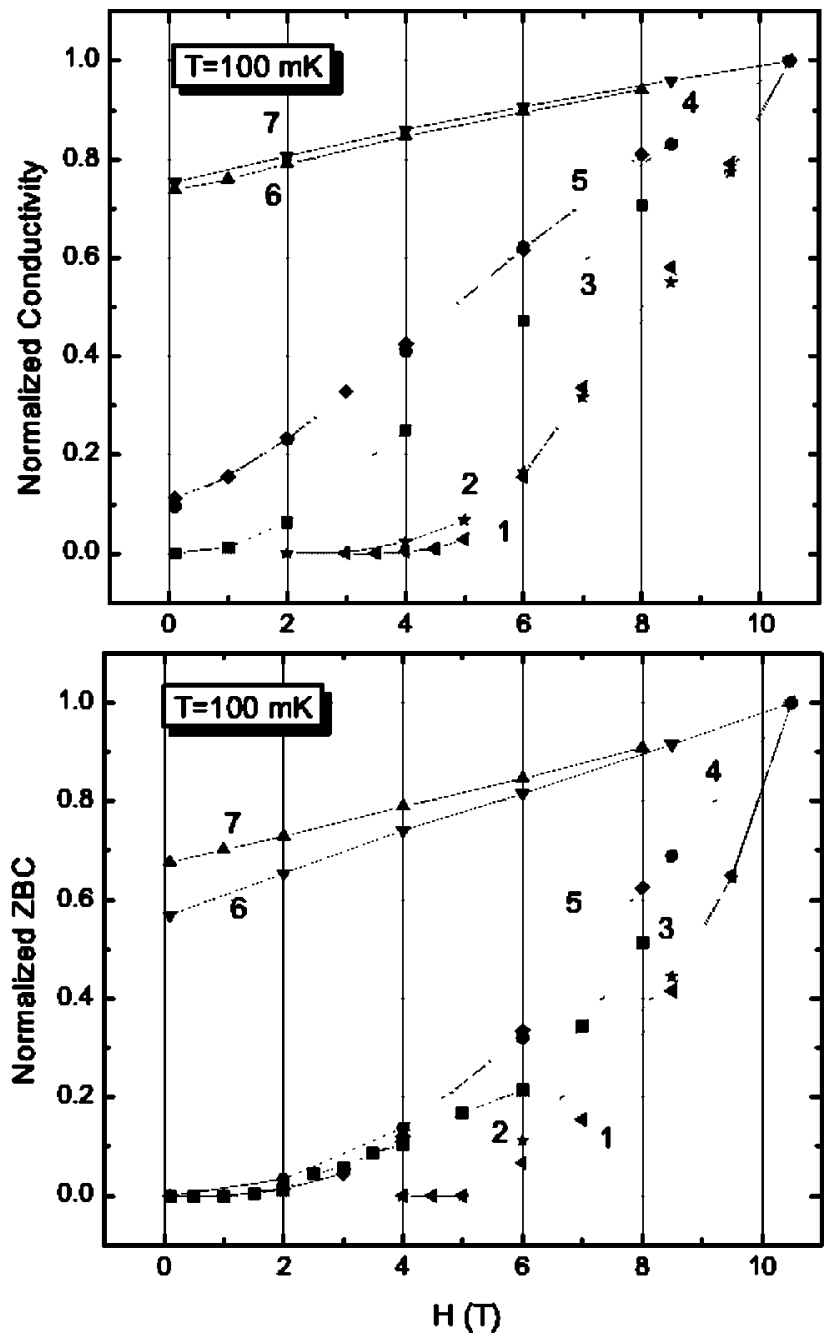

FIG. 7. Normalized conductivity and zero bias conductance vs $H$ for all samples at $T=100 \mathrm{mK}$. Zero bias conductance for each sample has been also rescaled by a numerical factor.

lating behavior, manifested as the vanishing tunneling zero bias conductance, seems to persist well into the metallic regime. At higher fields, the tunneling ZBC grows approximately as $H^{2}$, and the transport conductivity increases linearly with field, as shown before. Metallic samples No. 6 and 7 are characterized by nonzero values of both conductivity and $\mathrm{ZBC}$ at $H=0.1 \mathrm{~T}$ and a slightly sublinear increase of both of these quantities with increasing field.

Observation of vanishing zero bias tunneling conductance, i.e., the vanishing density of extended states at the Fermi level, at fields where the transport conductivity is already metallic, raises concerns over the possibility of inhomogeneities in the samples. However, the gradual and consistent variation of the $d I / d V$ curves of several different samples and of their $N(0)$ values gives us confidence that the observed effect is intrinsic and is not due to the imperfections in the GdSi film. Samples No. 1-4 have been made during the same fabrication run and are expected to have a slight gradient in the carrier concentration due to each sample's proximity to either gadolinium or silicon evaporation source, with the conductivity increasing from No. 1 to No. 4.
The observed variations in the conductivity and tunneling conductance are subtle and consistent with the sample's position in the evaporation chamber, therefore we conclude that the macroscopic inhomogeneities do not dominate the transport properties of the GdSi films. The absence of Gd clustering has been also verified by the XAFS studies. ${ }^{18}$

The results of our experiments can be summarized as follows. The shape of the DOS evolves gradually and continuously with increasing magnetic field from insulating to metallic. The Coulomb correlation gap, associated with the insulating behavior, shrinks as the field increases. At the same fields, the transport experiments on the sample already show metallic transport conductivity. Zero bias tunneling conductance grows as a strong (quadratic) function of the applied field. For samples very close to the transition, one can observe a situation where the tunneling conductance is vanishingly small, and the transport conductivity is appreciable. This is a result we did not expect, as from earlier results ${ }^{17}$ we anticipated that at the transition $N(E)$ and $\sigma_{0}$ would vanish together. The detailed study of the samples closer to the critical regime than the samples in our previous work indicate that this is not the case. Based on these results, we conclude that the vanishing zero bias tunneling conductance and superlinear $d I / d V(V)$, usually associated with the insulating behavior, persist well into the metallic regime.

\section{DISCUSSION}

Observation of a measurable metallic conductivity simultaneously with an apparent absence of the extended states at the Fermi level as determined by tunneling spectroscopy appears paradoxical at first sight. Nevertheless, a situation where this is possible can be easily pictured. Consider a strongly disordered system approaching the MIT from an insulating side. When a small fraction of all carriers becomes delocalized at the transition, the current may flow via a very small number of conduction paths among the disordered sites. The conductivity due to these paths is much higher than that due to the rest of the carriers, which are still localized and participate in transport by the thermally activated variable-range hopping. At low temperature the contribution of the localized carriers to the conductivity is exponentially small compared to that of the extended states. The overall conductivity therefore will be determined by the few high mobility extended carriers. On the other hand, the DOS that is observed in an experiment, such as ours, reflects an average number among all microscopic states in the sample at a given energy, thus its magnitude is determined by the dominating species. In this regime of atomic inhomogeneity, transport and tunneling measure two different quantities, and this is seen in our experiments. Near the transition, a small number of carriers have a metallic DOS with $N(E) \sim E^{1 / 2}$ and $N(0) \neq 0$, however, the vast majority of states show an insulating type of DOS with a parabolic energy dependence and a vanishing $N(0)$. When summed over all microscopic states with appropriate weights proportional to the numbers of each species, the contribution of the localized states to the DOS overwhelmingly prevails over that of the metallic ones, and as a result, at the transition the total DOS appears to be 
insulating. In this case the DOS at $E=0$ at the MIT is likely to vanish. As the sample is driven towards the metallic regime and the number of the extended carriers grows, the DOS acquires an intermediate shape, i.e., it shows features due to both insulating and metallic species simultaneously. Gradually the signatures of the metallic behavior begin to take over a larger and larger range of energy, until eventually they dominate the whole DOS. While the sample is tuned into the metallic regime, its conductivity rapidly increases and exhibits metallic behavior. Therefore it is plausible that in the same conditions, the transport experiments will place this sample on the metallic side of the transition, while in the tunneling measurements it will appear as an insulator.

The results of these current experiments are, nevertheless, unexpected. Our findings lead us to speculate that on this scale the material might be inhomogeneous, either intrinsically or because of the natural atomic scale inhomogeneities of a doped material. In either case, it signals to us that our previous way of thinking of the MIT in terms of diverging length scales may be incorrect and atomic scale inhomogeneities may dominate instead. The spontaneous formation of inhomogeneous regions near phase transitions has been used to describe various systems in critical regimes, such as manganites. ${ }^{29} \mathrm{We}$ do not have strong opinions whether this could be the case here or whether we observe the consequences of the natural inhomogeneity on the atomic scale associated with a $14 \%$ concentration of gadolinium in amorphous silicon. We do note, however, that a similar inhomogeneity model was used to understand the MIT in Si:P and $\mathrm{Si}: \mathrm{B}^{30,32}$

It could be argued that this description is a percolation transition at an atomic scale and that a percolation threshold in a two component system in three dimensions should occur at a fractional concentration of $x_{c} \sim 0.43$ (for site percolation in a diamond structure) ${ }^{31}$ However, this transition must take into account in addition to the natural inhomogeneity at the atomic scale, the strong correlations that are ignored in a classical model. Indeed, while we resort to a single particle picture of mobility and density of states for descriptive reasons, this transition is believed to be driven by the correlations which are not single particle effects.

\section{ACKNOWLEDGMENTS}

We thank S. Kivelson, O. Naaman, and A. Frydman for valuable discussions. This work was supported by the NSF Grant No. DMR-0097242.
${ }^{1}$ P. A. Lee and T. V. Ramakrishnan, Rev. Mod. Phys. 57, 287 (1985); B. Kramer and A. McKinnon, Rep. Prog. Phys. 56, 1469 (1993); N. F. Mott, Conduction in Non-Crystalline Materials (Clarendon Press, Oxford, 1993).

${ }^{2}$ B. L. Altshuler and A. G. Aronov, JETP Lett. 30, 514 (1979); B. L. Altshuler, D. Khmel'nitzkii, A. I. Larkin, and P. A. Lee, Phys. Rev. B 22, 5142 (1980).

${ }^{3}$ N. F. Mott, J. Non-Cryst. Solids 1, 1 (1968).

${ }^{4}$ A. L. Efros and B. L. Shklovskii, J. Phys. C 8, L49 (1975).

${ }^{5}$ B. L. Altshuler and A. G. Aronov, Solid State Commun. 30, 115 (1979); Sov. Phys. JETP 50, 968 (1979).

${ }^{6}$ W. L. McMillan, Phys. Rev. B 24, 2739 (1981).

${ }^{7}$ B. L. Shklovskii and A. L. Efros, Fiz. Tverd. Tela (S.-Petersburg) 14, 825 (1980) [Sov. Phys. Semicond. 14, 487 (1980)].

${ }^{8}$ J. G. Massey and M. Lee, Phys. Rev. Lett. 75, 4266 (1995); 77, 3399 (1996); 79, 3986 (1997); M. Lee, J. G. Massey, V. L. Nguyen, and B. I. Shklovskii, Phys. Rev. B 60, 1582 (1999); J. G. Massey and M. Lee, ibid. 62, R13270 (2000).

${ }^{9}$ G. Hertel, D. J. Bishop, E. G. Spencer, J. M. Rowell, and R. C. Dynes, Phys. Rev. Lett. 50, 743 (1983); D. J. Bishop, E. G. Spencer, and R. C. Dynes, Solid-State Electron. 28, 73 (1985); H.-L. Lee, J. P. Carini, D. V. Baxter, and G. Grüner, Phys. Rev. Lett. 80, 4261 (1998); H.-L. Lee, J. P. Carini, D. V. Baxter, W. Henderson, and G. Grüner, Science 287, 633 (2000); E. Helgren, G. Grüner, M. R. Ciofalo, D. V. Baxter, and J. P. Carini, Phys. Rev. Lett. 87, 116602 (2001).

${ }^{10}$ A. F. Ioffe and A. R. Regel, Prog. Semicond. 4, 237 (1960).

${ }^{11}$ T. F. Rosenbaum, K. Andres, G. A. Thomas, and R. N. Bhatt, Phys. Rev. Lett. 45, 1723 (1980); B. W. Dodson, W. L. McMillan, J. M. Mochel, and R. C. Dynes, ibid. 46, 46 (1981); W. N. Shafarman, D. W. Koon, and T. G. Castner, Phys. Rev. B 40,
1216 (1989).

${ }^{12}$ S. von Molnar and S. Methfessel, J. Appl. Phys. 38, 959 (1967); S. von Molnar, A. Briggs, J. Flouquet, and G. Remenyi, Phys. Rev. Lett. 51, 706 (1983); S. Washburn, R. A. Webb, S. von Molnar, F. Holtzberg, J. Flouquet, and G. Remenyi, Phys. Rev. B 30, 6224 (1984); J. Jaroszynski and T. Dietl, Physica B 177, 469 (1992).

${ }^{13}$ M. A. Paalanen, T. F. Rosenbaum, G. A. Thomas, and R. N. Bhatt, Phys. Rev. Lett. 48, 1284 (1982); S. Waffenschmidt, C. Pfleiderer, and H. v. Löhneysen, ibid. 83, 3005 (1999).

${ }^{14}$ I. Terry, T. Penney, S. von Molnar, J. M. Rigotty, and P. Becla, Solid State Commun. 84, 235 (1992); C. Leighton, I. Terry, and P. Becla, Europhys. Lett. 42, 67 (1998); Phys. Rev. B 58, 9773 (1998).

${ }^{15}$ F. Hellman, M. Q. Tran, A. E. Gebala, E. M. Wilcox, and R. C. Dynes, Phys. Rev. Lett. 77, 4652 (1996).

${ }^{16}$ M. Liu and F. Hellman, Phys. Rev. B 67, 054401 (2003).

${ }^{17}$ W. Teizer, F. Hellman, and R. C. Dynes, Phys. Rev. Lett. 85, 848 (2000).

${ }^{18}$ F. Hellman, W. Geerts, and B. Donehew, Phys. Rev. B 67, 012406 (2003).

${ }^{19}$ W. Teizer, F. Hellman, and R. C. Dynes, Solid State Commun. 114, 81 (2000).

${ }^{20}$ P. Xiong, B. L. Zink, S. I. Applebaum, F. Hellman, and R. C. Dynes, Phys. Rev. B 59, R3929 (1999).

${ }^{21}$ W. Teizer, F. Hellman, and R. C. Dynes, in Proceedings of the 25th International Conference on the Physics of Semiconductors, Osaka, 2000, edited by N. Miura and T. Ando (Springer, New York, 2000).

${ }^{22}$ B. L. Zink, E. Janod, K. Allen, and F. Hellman, Phys. Rev. Lett. 83, 2266 (1999). 
${ }^{23}$ F. Hellman, D. R. Queen, R. M. Potok, and B. L. Zink, Phys. Rev. Lett. 84, 5411 (2000).

${ }^{24}$ D. N. Basov, A. M. Bratkovsky, P. F. Henning, B. Zink, F. Hellman, C. C. Homes, and M. Strongin, Europhys. Lett. 57, 240 (2002).

${ }^{25}$ W. Teizer, F. Hellman, and R. C. Dynes, Int. J. Mod. Phys. B 17, 3723 (2003); (unpublished).

${ }^{26}$ W. Teizer, F. Hellman, and R. C. Dynes, Phys. Rev. B 67, R121102 (2003).

${ }^{27}$ A. M. Finkelstein, Sov. Phys. JETP 59, 212 (1984).
${ }^{28}$ C. Castellani, C. Di Castro, P. A. Lee, and M. Ma, Phys. Rev. B 30, 527 (1984).

${ }^{29}$ E. Dagotto, T. Hotta, and A. Moreo, Phys. Rep. 344, 1 (2001); T. Becker, C. Streng, Y. Luo, V. Moshnyaga, B. Damaschke, N. Shannon, and K. Samwer, Phys. Rev. Lett. 89, 237203 (2002).

${ }^{30}$ S. Wagner, M. Lakner, and H. v. Löhneysen, Phys. Rev. B 55, 4219 (1997).

${ }^{31}$ R. Zallen, The Physics of Amorphous Solids (Wiley, New York, 1983), p. 170.

${ }^{32}$ T. G. Castner, Phys. Rev. B 68, 115201 (2003). 